\title{
Time-Series Analysis of COVID-19 in Iran: A Remote Sensing Perspective
}

\author{
Nadia Abbaszadeh Tehrani, Abolfazl Mollalo, Farinaz Farhanj, Nooshin \\ Pahlevanzadeh and Milad Janalipour
}

This chapter reports on a national study of COVID-19 using remote sensing (RS) indicators in Iran. Time-series analysis is performed on RS indicators ( $n=12)$ including wind speed, temperature, evaporation, carbon monoxide (CO), nitrogen dioxide $\left(\mathrm{NO}_{2}\right)$, Sulphur dioxide $\left(\mathrm{SO}_{2}\right)$, ozone $\left(\mathrm{O}_{3}\right)$, formaldehyde ( $\left.\mathrm{HCHO}\right)$, cloud cover, precipitation, air pressure and soil moisture (SM) to identify remotely sensed products that may contribute to COVID-19 transmission. Mann-Kendall test is employed to summarize time-series observations. Further, a correlation analysis is performed between Z-scores obtained from the Mann-Kendall test and the number of COVID-19 cases. Findings indicated that the precipitation, $\mathrm{NO}_{2}$, and $\mathrm{SO}_{2}$ have high correlations with number of COVID-19 cases with Spearman correlation coefficient of -0.39, -0.33, and -0.31, respectively. Findings may provide useful insights for public health decision makers by improving the accuracy of predicative models.

\subsection{Introduction}

As of 1 July 2020, Iran has been identified as one of the top ten countries with the highest number of reported COVID-19 cases. Several neighboring countries such as Bahrain, Iraq, Georgia, Kuwait, Oman, Afghanistan, Lebanon, and Pakistan reported that their first cases of COVID-19 was imported from passengers traveling from Iran [1]. As of 3 September 2020, over 381,000 confirmed cases and almost 22,000 deaths had been reported from Iran. However, the real figure is largely underestimated [2]. It is predicted that the country will face several waves of the pandemic due to ineffective controlling strategies such as early reopening and ease of restrictions.

Several studies have identified associations between environmental indicators and COVID-19 transmission. For instance, in China, Yongji et al. (2020) examined the relationship between ambient air pollution and daily (confirmed) COVID-19 cases using generalized additive models. They found a positive association between COVID-19 and particulate matter 2.5, carbon monoxide, nitrogen dioxide, and ozone, while Sulphur dioxide was negatively associated with the disease [3]. Ma et al. (2020) modeled the relationship between daily COVID-19 mortalities and temperature and relative humidity variations using time-series analysis. They found a positive association with temperature and negative association with relative humidity [4].

Epidemiological investigations of infectious diseases mostly concentrate on medical aspects and infection control and disregard the geographic components of the diseases [5-7]. Geospatial technologies such as Remote Sensing (RS) and geographic information system (GIS) have been identified useful in monitoring a variety of infectious diseases when they are coupled with 
data-driven techniques [8,9]. GIS and RS have been utilized in the study of COVID-19 across the world. For instance, Liu et al. (2020) used RS data such as nighttime light and air quality index to assess the impact of COVID-19 lockdown on human lives in Mainland, China. Their results suggested that with the implementation of lockdown policies, the nighttime light radiances generally decreased in the entire Mainland, and a significant decline was observed in commercial center regions. Meanwhile, air quality significantly improved [10]. In a GIS-based study in the United States, Mollalo et al., (2020) utilized multi-scale geographically weighted regression to explain the variations of COVID-19 incidence at the county level across the country. They compiled a geodatabase of 35 explanatory variables, including environmental, behavioral, and socio-economic factors. Their results indicated that socio-economic variables, particularly income inequality, could explain more than $68 \%$ of variations of disease incidence compared to environmental factors $[11,12]$.

To our knowledge, there are limited studies that have utilized remotely sensed data to monitor COVID-19 in any region of Iran, especially at the national level. To bridge the gap, we examined the applicability of RS coupled with time-series analysis in Iran as our study area.

\subsection{Materials and Methods}

In this section, we describe the study area, data used and methodology for identifying time-series relation among COVID-19 and environmental indicators obtained from satellite observations.

\subsubsection{Study Area}

The study area covers Iran. This country with the area of $648,195 \mathrm{~km}^{2}$ is considered as the 17 th largest country in the world. Iran is divided into 31 provinces (Figure 22.1). Two types of datasets including the number of COVID-19 cases and remotely sensed data were compiled. Figure 22.1 shows the geographic location of study area together with the normalized number of COVID-19 cases by the population.

\subsubsection{Disease Dataset}

The number of COVID-19 cases in each province was obtained from the Ministry of Health and Medical Education. The data were only available for 22 provinces, excluding Alborz, Isfahan, Qom, Razavi Khorasan, Semnan, and Tehran provinces, which are shown in Figure 22.1. The actual number of cases is presented in NCD column of Table 22.2. The number of COVID-19 cases is registered from 20 February 2020 to 19 April 2020, which is about 53000 cases.

\subsubsection{Remotely Sensed Data}

In this study, various satellite data sources and products were obtained from Google Earth Engine (GEE) platform (https://earthengine.google.com/). GEE is an efficient cloud computing tool that provides georeferenced and calibrated RS data of a variety of satellite imagery [13]. It allows researchers and users to simultaneously process, visualize, and analyze time-series geospatial data in a simple and quick way [14]. GEE was utilized to provide 12 spatial indicators including wind speed, temperature, evaporation, carbon monoxide $(\mathrm{CO})$, nitrogen dioxide $\left(\mathrm{NO}_{2}\right)$, Sulphur dioxide $\left(\mathrm{SO}_{2}\right)$, ozone $\left(\mathrm{O}_{3}\right)$, formaldehyde $(\mathrm{HCHO})$, cloud cover, precipitation, surface pressure, and soil moisture (SM) (Table 22.1). The selection of parameters is based on literature review and available RS indicators $[3,4,10-12]$.

In order to extract time-series RS indicators $(n=12)$ from GEE, Terra Moderate Resolution Imaging Spectroradiometer (MODIS), Sentinel-5 Precursor (Sentinel-5P), Global Precipitation Measurement (GPM), Soil Moisture Active Passive (SMAP), National Centers for Environmental Prediction (NCEP) Climate Forecast System Reanalysis (CFSR), and Global Land Data 


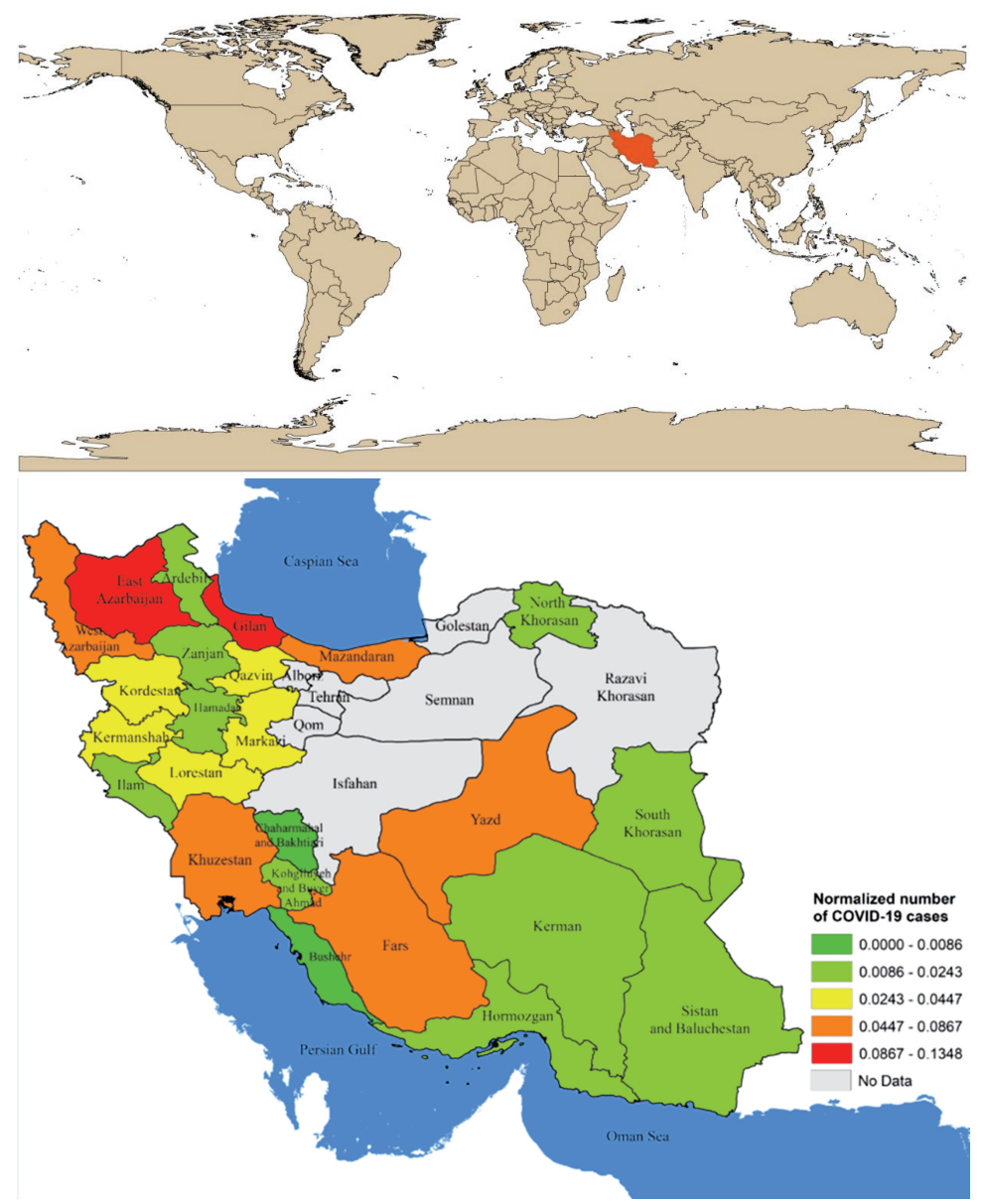

\section{FIGURE 22.1}

The geographic location of Iran in world map, and the normalized number of COVID-19 cases obtained until 19 April 2020 for each province

Assimilation System (GLDAS) were used. These data were acquired from February 20, 2020 (starting date of COVID-19 outbreak in Iran) to 19 April 2020.

\subsubsection{MODIS Data}

According to previous studies, the number of COVID-19 cases may be influenced by the changes of temperature and evaporation $[15,16]$. Terra MODIS MOD11A1 Collection 6 (C6) product provides daily daytime, and nighttime land surface temperature (LST) at a spatial resolution of $1 \mathrm{~km}$ [17]. We selected Terra MODIS data, because the atmosphere is more stable at the early hours of the day. Atmospheric instability can influence dispersion of aerosols, pollutants, and smoke plumes in some areas $[18,19]$. This product is vastly used and evaluated by many researchers across the world $[20,21]$. The MOD11A1 with scientific datasets (SDS) name of "LST-Day-1km" $\left({ }^{\circ} K\right)$ was obtained from GEE data catalog during the study period.

Terra MODIS level 4 MOD16A2 C6 product, which provides land surface total evapotranspiration $(\mathrm{ET})\left(\mathrm{kg} / \mathrm{m}^{2}\right)$ datasets in 8-day at $500 \mathrm{~m}$ resolution, have also been used [22] ET product can be used to calculate regional water and energy balance. Provinces with high ET values may have high COVID-19 cases, therefore researchers believe that ET may be an effective indicator. Hence, change of ET and its effect on COVID-19 are investigated. The MOD16A2 product 
with SDS name of "ET-500m" was also downloaded from GEE data catalog. This product has been applied, evaluated, and validated by many researchers [23, 24].

\subsubsection{GPM Data}

Previous studies indicate that precipitation is an important indicator that may lead to an influence on microbial pollution which may effect on the number of COVID-19 cases [25]. For this reason, this indicator is incorporated in the dataset. GPM is an international earth's precipitation observation science mission that measures amounts of rainfall and snowfall $(\mathrm{mm} / \mathrm{hr})$ for every three hours at a spatial resolution of 0.1 arc degrees $(\approx 11.1 \mathrm{~km})$. GPM data products can improve analyzing climate data all over the world [26, 27]. The data were previously validated by some researchers [28-31]. GPM version 6 with SDS name of IR (Infrared) precipitation was utilized.

\subsubsection{NCEP CFSR Data}

Surface pressure (SP) is an indicator that effects some respiratory diseases such as chronic obstructive pulmonary disease, therefore it is employed in this study [32]. NCEP CFSR, one of the global reanalysis datasets, designed to compute an estimation of the global interaction between atmosphere, ocean, sea ice, and land surface [33]. Surface pressure (Pa), albedo (\%), sea surface temperature $\left({ }^{\circ} K\right)$, soil temperature $\left({ }^{\circ} K\right)$, snow depth $(\mathrm{m})$, vegetation cover $(\%)$, relative humidity $(\%)$, planetary boundary layer height $(\mathrm{m})$, and surface roughness $(\mathrm{m})$ were available variables in the datasets at 0.2 arc degrees $(\approx 22.2 \mathrm{~km})$ resolution. These reanalysis datasets were widely used and evaluated in many studies [34, 35]. Among these, surface pressure with SDS name of "Pressure-surface" was the only variable used in this study.

\subsubsection{SMAP Data}

Soil Moisture (SM) is an environmental indicator that can be provided by RS data. Change of SM may influence COVID-19 cases. Hence, this indicator is included in this study. SMAP measures surface values and subsurface SM $(\mathrm{mm})$ every 3 days at 0.25 arc degrees $(\approx 27.75 \mathrm{~km})$ resolution with the combination of passive (radiometer) and active (radar) instruments [36]. Scientists can use SMAP data products to better investigate different environmental applications, such as drought monitoring, climate change analyzing, flood prediction, and monitoring of agricultural crop growth [37-39]. These data were evaluated and validated in some research projects [40, 41]. In this study, level 3 surface soil moisture (SSM) with SDS name of SSM was acquired.

\subsubsection{GLDAS Data}

Similar to surface pressure, wind speed can be an influential factor that may be associated with the transmission of respiratory diseases [42]. GLDAS utilizes different earth observation satellites and ground-based data. It is mainly used to generate wind speed $(\mathrm{m} / \mathrm{s})$, albedo $(\%)$, and soil temperature $\left({ }^{\circ} \mathrm{K}\right)$. These data are provided at 0.25 arc degrees $(\approx 27.75 \mathrm{~km})$ resolution every 3 hours, which are used and evaluated in many research projects, such as water resource management, drought monitoring, weather forecasting, and flux cycle studies [42, 43]. In this study, wind speed (WS) was extracted from GLDAS data products using GEE platform.

\subsubsection{Sentinel-5P Data}

Copernicus program provides some environmental parameters that can present appropriate information about diseases [44]. They can show crowded and industrial areas that people have a high interaction. Hence, it is employed to extract some environmental indicators and the their effects on COVID-19. Sentinel-5P sensor called TROPOspheric Monitoring Instrument (Tropomi) is designed to monitor the atmosphere, climate, air quality, and solar radiation, at a spatial resolution of 0.01 arc degrees $(\approx 1.11 \mathrm{~km})$, and a spectral range of $(270-495),(675-775)$, and $(2305-2385) \mathrm{nm}$ [45]. In this study, Near Real-Time (NRTI) air pollutant concentrations, including CO, NO2, SO2, $\mathrm{O} 3$, and $\mathrm{HCHO}$ were obtained. Also, cloud cover fraction data among NRTI level 3 cloud products of Sentinel-5P was extracted from GEE data catalog and utilized as explanatory variable. 
TABLE 22.1

Remotely sensed data used in this study together with spatial resolution and sources.

\begin{tabular}{llcc}
\hline Source & Indicator & Spatial resolution & Citation \\
MODIS & LST & $1 \mathrm{~km}$ & {$[15-17,46]$} \\
& Evaporation & $500 \mathrm{~m}$ & \\
GPM & Precipitation & $11 \mathrm{~km}$ & {$[47]$} \\
NCEP CFSR & Surface Pressure & $22 \mathrm{~km}$ & {$[32]$} \\
SMAP & Soil Moisture & $27 \mathrm{~km}$ & {$[40,41,48]$} \\
GLDAS & Wind Speed & $27 \mathrm{~km}$ & {$[32,49]$} \\
Sentinel-5P & $\mathrm{CO}, \mathrm{NO}_{2}, \mathrm{SO}_{2}, \mathrm{O}_{3}$, & $1 \mathrm{~km}$ & {$[44]$} \\
& $\mathrm{HCHO}, \mathrm{Cloud}$ Cover & & \\
\hline
\end{tabular}

\subsubsection{Methodology}

In the proposed method, time-series analysis of 12 spatial indicators including wind speed, temperature, evaporation, $\mathrm{NO}_{2}, \mathrm{SO}_{2}, \mathrm{CO}, \mathrm{O}_{3}$, cloud cover, $\mathrm{HCHO}$, precipitation, surface pressure and SM obtained from GLDAS, MODIS, Sentinel-5P, GPM, NCEP CFSR, and SMAP satellites/datasets. Pre-processing tasks including some scale factors were performed. Further, the average of each indicator was estimated. Mann-Kendall test was employed to produce Z score maps of time-series observations for each indicator. Mann-Kendall test was used to indicate the general trend of a variable resulted from the processing of time series data. Finally, correlation between $\mathrm{Z}$ values and the number of COVID-19 cases was calculated to identify the most effective indicators. Figure 22.2 depicts the workflow of the research.

\subsubsection{Mann-Kendall Test for time-series Analysis}

Mann-Kendall test was utilized to study time-series changes of the selected environmental RS indicators. The main advantage of this test is that it is not influenced by observations with the drastic changes [50, 51]. Mann-Kendall test was also employed for two main reasons: 1- To study severe time-series and changes of spatial indicators in the study area, 2- since the number of registered COVID-19 cases have been registered in period of two months, we need to make a parameter from daily and weekly satellite observations to compare it with the number of COVID-19 cases. Z score of Mann-Kendall test provides a change of an indicator in period of two months, therefore it is possible to perform a correlation analysis among them.

Suppose $n$ observations in accordance with $x_{1}, x_{2}, \ldots, x_{n}$ in the Mann-Kendall test, $\mathrm{S}$ variable is calculated based on Equation (22.1):

$$
\mathrm{S}=\sum_{k=1}^{n-1} \sum_{j=k+1}^{n} \operatorname{sgn}\left(x_{j}-x_{k}\right)
$$

where, sgn ( $\mathrm{x})$ is calculated as:

$$
\operatorname{sgn}(x)= \begin{cases}1 & \text { if } x>0 \\ 0 & \text { if } x=0 \\ -1 & \text { if } x<0\end{cases}
$$

The variance of $\mathrm{S}$ is calculated as:

$$
\operatorname{var}(\mathrm{S})=\frac{\left.\left[n(n-1)(2 n+5)-\sum_{i=0}^{m}\left(t_{i}\left(t_{i}-1\right)\left(2 t_{i}+5\right)\right)\right)\right]}{18}
$$

where $\mathrm{m}$ is the number of groups with similar values and $t_{i}$ is the number of points in group $\mathrm{i}$. After calculating the variance, the $\mathrm{Z}$ score is calculated using the Equation (22.4). Positive Z scores indicate positive changes in the variable trend, while negative $\mathrm{Z}$ scores indicate decreasing trend of the studied variable. Values greater than 1.96 and smaller than -1.96 indicate significant changes 


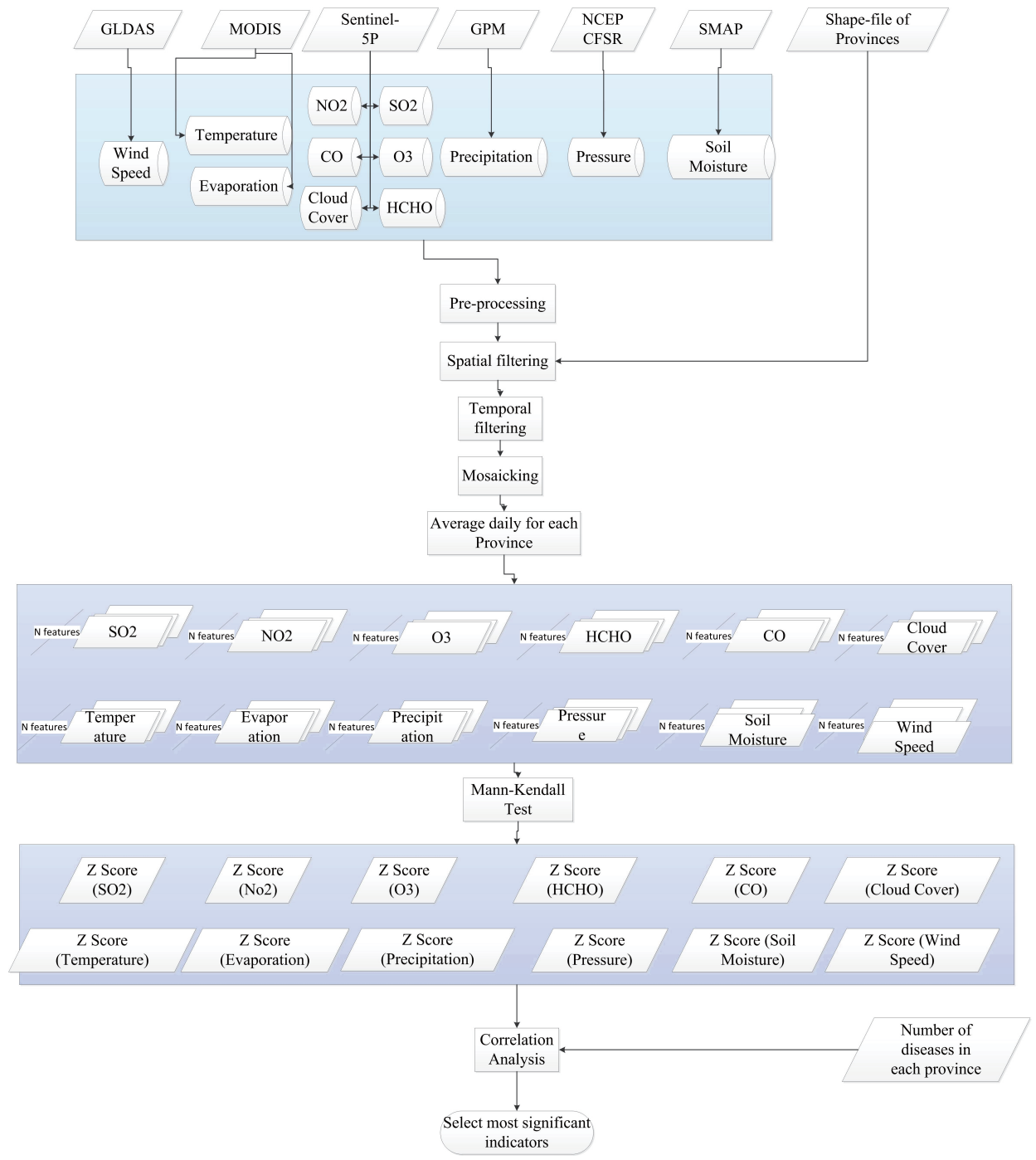

\section{FIGURE 22.2}

Workflow of the proposed method to identify effective time-series RS indicators on COVID-19

at $95 \%$ confidence level.

$$
Z S \text { core }= \begin{cases}\frac{(S-1)}{\sqrt{\operatorname{Var}(S)}} & \text { if } S>0 \\ 0 & \text { if } S=0 \\ \frac{(S+1)}{\sqrt{\operatorname{Var}(S)}} & \text { if } S<0\end{cases}
$$

\subsubsection{Correlation Analysis for Validation}

The relation between COVID-19 and spatial indicators was investigated using correlation analysis. Correlation analysis was examined between $\mathrm{Z}$ score of each indicator and the number of COVID-19 cases. Three correlation coefficients including Pearson, Spearman, and Kendall were utilized, which were frequently used in previous studies [52]. If at least two correlation methods confirm high correlation values, it can be concluded the spatial indicator may be related to the COVID-19.

Pearson is a linear approach to measure correlation among two variables, which was frequently 
used in previous researches. The range of values in Pearson is changing between -1 and 1 . Values approaching -1 and 1 show high correlation between variables [53]. Spearman is another correlation estimation method which assesses monotonic relationships between variables [54]. The Kendall rank correlation coefficient is another way to measure correlation of two variables [55]. To assess the significance level of the three correlation approaches, P-values were also computed.

\subsection{Results and Discussion}

Figure 22.3 shows the map of average time-series of each indicator across Iran. According to cloud cover map, maximum cloud was observed in North and West regions of Iran. Maximum values of $\mathrm{CO}$ were seen in regions near Caspian Sea, Persian Gulf and Oman Sea. While, minimum values were observed in central areas. Precipitation of the wettest regions in Iran was higher than other regions. While, precipitation of central and east regions were lower than other regions. Maximum precipitation was occurred in south regions of Iran, where some flood events were also reported during 2020. HCHO over Tehran, Gilan, Mazandaran, Khuzestan, and Bushehr provinces was more than others during the study period. Temperature of north and west regions of Iran was lower than south and east, which is true based on climate of those areas. Wind speed of east regions was more than north and west ones during the study period.

Maximum value of $\mathrm{NO}_{2}$ was observed in Gilan, Mazandaran, Albourz, Tehran, Qom, Markazi, Isfahan, and Khuzestan. High density population in the mentioned areas may be a possible explanation for this finding. Moreover, a high number of COVID-19 cases were registered in the mentioned provinces. Time-series analysis of $\mathrm{O}_{3}$ showed a higher density in higher longitude compared to lower altitudes.

According to time-series analysis, the larger amount of $\mathrm{SO}_{2}$ values were observed in Gilan, Mazandaran, Tehran, Khuzestan, Bushehr, East Azarbaijan, and Kerman provinces. Surface pressure of south, west and central regions of Iran was more than other areas. In addition, SM of north and west regions of Iran was more than other areas, while central regions had the lowest values compare with the other regions.

The $\mathrm{Z}$ scores of each indicator obtained from Mann-Kendall test were presented in Table 22.3. Cloud cover time-series analysis shows that the percentage of cloud cover in south and north regions including Alborz, Isfahan, Fars, Hormozgan, Semnan, Sistan and Baluchestan, and Yazd have been significantly increased during study period $(\mathrm{Z}$ score $<1.96)$. While, a significant decrease with Z Score lower than -1.96 in cloud cover was observed over Kordestan and West Azarbaijan (Wazar). Among the mentioned provinces, Alborz, Isfahan, Semnan and Yazd have a high number of COVID-19 cases. In general, according to Z score results, it seems that the cloud cover is not an effective indicator to find provinces with low and high numbers of COVID-19 cases were not detected.

Although the amount of evaporations over all provinces has been increased during the study period, it is not significant $(0<\mathrm{Z}$ score $<1.96)$. Therefore, it seems that ET is not a related indicator to COVID-19.

According to satellite observations and Mann-Kendall test, precipitation was significantly increased over Bushehr, Isfahan, Fars, Hormozgan, Kerman, Kohgiluyeh and Buyer Ahmad, North Khorasan (Nkhorsan), Razavi Khorasan (Rkhorasan), Semnan, Sistan and Bluchestan, and South Khorasan (Skhorasan). During the study period, the number of COVID-19 diseases in the mentioned provinces was lower than others. It seems that $\mathrm{Z}$ score of precipitation is negatively correlated with the number of COVID-19 cases.

$\mathrm{Z}$ score of temperature over all provinces was positive and greater than 1.96 , which shows an increase in temperature in all provinces. Since the research period was between winter and spring, the results seem to be correct. There was no significant association between the number of COVID-19 cases and surface pressure and the association has not changed over majority of provinces. Based on SMAP observations, SM of central and east regions of Iran i.e., Fras, Kerman, Razavi Khorasan, South Khorasan, and Yazd were increased. Moreover, the Z scores of west regions 


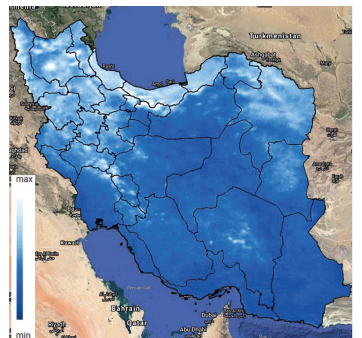

(a)

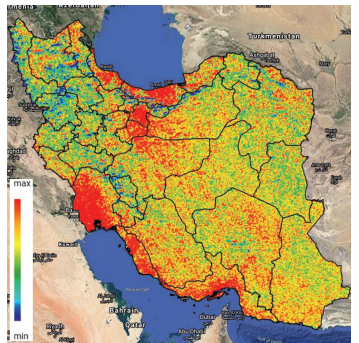

(d)

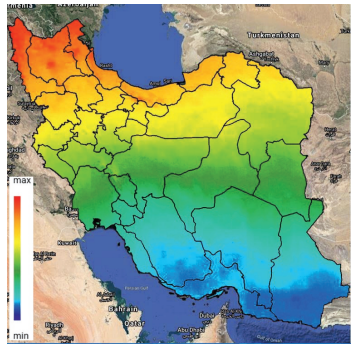

(g)

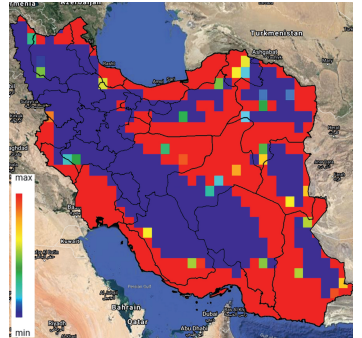

(j)

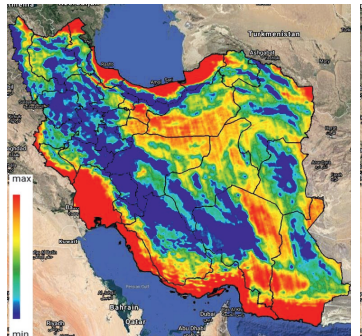

(b)

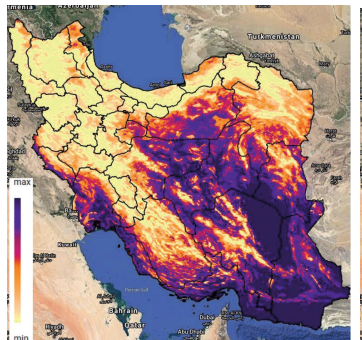

(e)

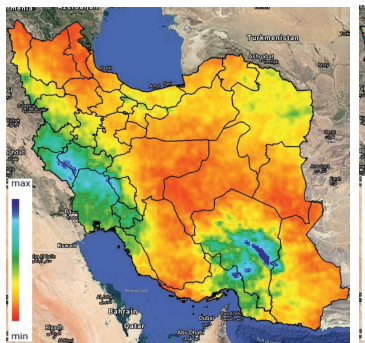

(h)

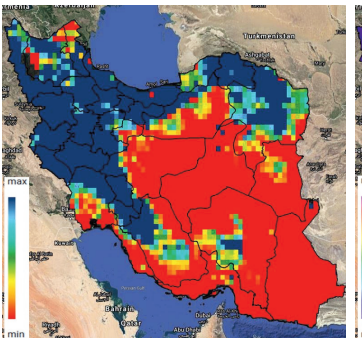

(k)

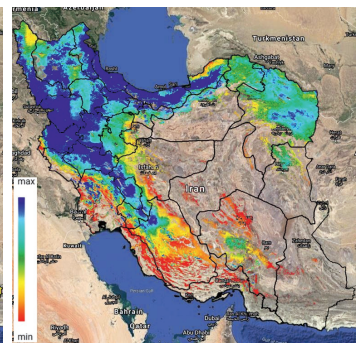

(c)

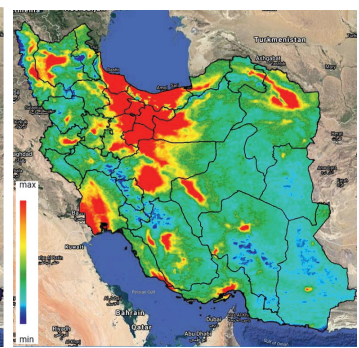

(f)

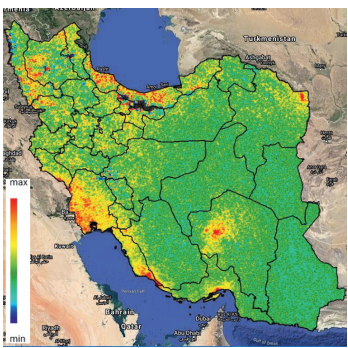

(i)

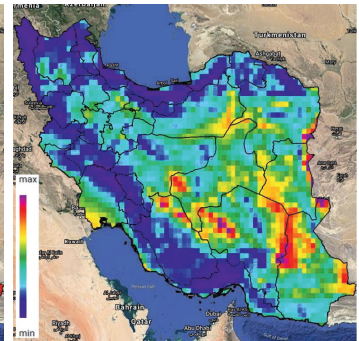

(1)

\section{FIGURE 22.3}

Average maps of indicators obtained from Iran in time of study(a) Cloud cover (b) CO (c) ET (d) HCHO (e) LST (f) NO2 (g) O3 (h) Precipitation (i) SO2 (j) Surface pressure (k) SM (l) Wind speed 
of Iran i.e., Ilam, Kermanshah, Khuzestan, Kordestan, Lorestan, Mazandaran, and Zanjan were decreased. According to the results, a strong relation among SM and the number of COVID-19 cases is not observed.

The values of $\mathrm{CO}, \mathrm{O} 3$ and $\mathrm{HCHO}$ indicators in all provinces were increasing, decreasing and increasing, respectively. NO2 was decreased in some provinces, especially in regions that the number of patients was higher than others. Moreover, based on the Z score, SO2 was decreased over all provinces. Due to several limitations of government on industries, universities, schools, and transportation, it appears that the results of NO2 and SO2 were reasonable. Since results of these indicators are complex, we use correlation analysis to find relation among these two indicators and COVID-19.

All the correlation coefficients (i.e., Pearson, Kendall, and Spearman) between spatial parameters and the number of COVID-19 cases, were calculated and presented in Table 22.3. A significant correlation is not observed between number of COVID-19 diseases and spatial indicators including evaporation, cloud cover, SP, SM, temperature, wind, $\mathrm{CO}$, formaldehyde, and $\mathrm{O} 3$ ( $\mathrm{P}$ value $>0.05)$. Their correlation values were closer to zero with large $\mathrm{P}$ values.

Unlike the study of Yongjian [3] that showed a positive correlation among CO, O3, and the number of COVID-19 cases, the correlation values of Pearson, Kendall, and Spearman for CO and $\mathrm{O} 3$ were $(0.04,0.06,0.09)$ and $(0.24,0.02,0.04)$, respectively. This suggests that there is no correlation among the mentioned indicators and the number of COVID-19 cases in Iran.

Based on outcomes of [4], a high correlation among humidity, temperature, and number of death due to COVID-19 is observed. In this study, Kendall and Spearman correlation values for ET are 0.19 and 0.24 , respectively, which shows a correlation among them, but it is not significant enough. Also, a low correlation value $(<0.1)$ is obtained between temperature and number of COVID-19 cases in this study.

According to our findings, precipitation, $\mathrm{NO} 2$, and $\mathrm{SO} 2$ are the most effective indicators that were highly correlated with the number of COVID-19 cases. Correlation values of Pearson, Kendall, and Spearman for precipitation were $-0.35,-0.28,-0.39$, respectively. Based on low $\mathrm{P}$-values of each correlation value $(\mathrm{P}-\mathrm{P}$ value $=0.08, \mathrm{~K}-\mathrm{P}$ value $=0.05, \mathrm{~S}-\mathrm{P}$ value $=0.05)$, it $\mathrm{can}$ be deduced that these associations are not due to the chance alone. Moreover, the $\mathrm{P}$ values of two correlation analysis methods confirmed that the correlation between precipitation and COVID-19 cases with confidence level of $95 \%$ is meaningful. Moreover, NO2 with the correlation values of $-0.25,-0.24$, -0.33 for Pearson, Kendall, and Spearman is another important indicator. Likewise, SO2 seems to be associated with the number of COVID-19 cases. P-values of NO2 and SO2 are higher than precipitation. Based on P-values of Kendall and Spearman, results with a confidence level of $85 \%$ are acceptable. This suggests that Kendall and Spearman correlation analysis methods confirm a significant correlation among NO2, SO2, and number of COVID-19 cases. Results of [3] are in agreement with our findings about $\mathrm{NO} 2$ and $\mathrm{SO} 2$.

Although this is a new study about the effect of RS indicators on COVID-19 in Iran, there are some limitations that should be considered. As the spatial and temporal resolutions of the used indicators are inconsistent, the integration of those indicators in data level would be a great limitation. Moreover, there are some no-data pixels in products of some days that should be corrected by interpolation methods. Another limitation is that the used products such as $\mathrm{SO} 2$ and NO2 should be calibrated based on ground observations for Iran to achieve more reliable outcomes. Finally, we employed the number of registered COVID-19 cases for two months. Since satellite observations can be provided on a daily basis, the use of daily statistics regarding COVID-19 cases can help us to perform a more robust validation on environmental indicators. The most important limitation is related to lack of detailed understanding of the nature of COVID individual cases \& deaths, or correcting for epidemiological \& personal health issues. 
TABLE 22.2

The $\mathrm{Z}$ scores of spatial indicators over all provinces

\begin{tabular}{|c|c|c|c|c|c|c|c|c|c|c|c|c|c|}
\hline $\begin{array}{l}\text { Province / } \\
\text { Indicators }\end{array}$ & \begin{tabular}{l}
$\Omega$ \\
$\Omega$ \\
$\vdots$ \\
0 \\
$\vdots$ \\
$\vdots$ \\
8 \\
\hdashline
\end{tabular} & 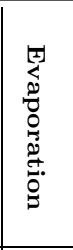 & 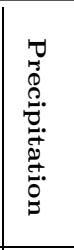 & 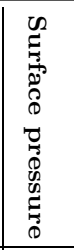 & 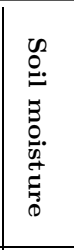 & 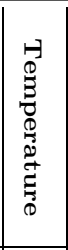 & \begin{tabular}{l}
\multirow{3}{0}{} \\
$\vdots$ \\
0 \\
$\mathbb{0}$ \\
$\mathbb{0}$ \\
0
\end{tabular} & ¿ & @ָ & $\begin{array}{l}Z \\
\text { O } \\
\text { N }\end{array}$ & O & రి & $\begin{array}{l}z \\
\Theta \\
\Theta\end{array}$ \\
\hline Alborz & 2.00 & 1.22 & 0.36 & -0.37 & 0.53 & 4.53 & -1.22 & 0.01 & -2.35 & -4.37 & 4.37 & -1.83 & $\mathrm{~N} / \mathrm{A}$ \\
\hline Ardebil & 0.84 & 0.73 & 0.19 & 0.09 & 0 & 1.82 & 1.08 & 2.60 & -1.67 & 2.48 & 5.31 & -3.61 & 0.018 \\
\hline Bushehr & 0.97 & -0.24 & 3.01 & -4.42 & 0.07 & 4.24 & 0.37 & 0.92 & 0.01 & -2.25 & 4.60 & -1.31 & 0.006 \\
\hline Ch-Mahal \& Bakh* & 1.48 & 1.22 & 0.76 & -0.25 & -1.28 & 2.81 & 0.47 & 4.30 & -1.88 & 1.95 & 5.12 & -0.31 & 0.008 \\
\hline East Azarbaijan & -0.53 & 1.22 & -0.38 & -0.79 & 1.51 & 5.16 & 2.07 & 3.52 & -2.21 & 0.01 & 4.53 & -3.56 & 0.111 \\
\hline Fars & 3.61 & 1.22 & 2.74 & -0.96 & 2.34 & 2.16 & 0.27 & 2.22 & -1.78 & \begin{tabular}{|l|}
-1.36 \\
\end{tabular} & 4.52 & -1.56 & 0.077 \\
\hline Gilan & -0.11 & 1.22 & -0.56 & 0.12 & -0.30 & 2.18 & 1.35 & 2.88 & -0.56 & -1.24 & 5.42 & -0.95 & 0.134 \\
\hline Golsetan & 1.71 & 1.71 & 0.22 & 0.08 & -1.11 & 1.62 & 0.03 & 2.31 & -1.16 & -0.20 & 4.40 & -2.80 & $\mathrm{~N} / \mathrm{A}$ \\
\hline Hamedan & -1.17 & 1.22 & -0.14 & -0.83 & -0.68 & 3.79 & 1.05 & 4.85 & -1.19 & -0.95 & 4.70 & -2.10 & 0.023 \\
\hline Hormozgan & 2.06 & 0.73 & 4.12 & -3.03 & 0.75 & 2.87 & 0.67 & 0.77 & -0.67 & -1.20 & 2.44 & -1.25 & 0.024 \\
\hline Ilam & 0.24 & 1.22 & 0.02 & -1.99 & -3.78 & 5.71 & -0.17 & 3.38 & -0.38 & \begin{tabular}{|l|}
0.56 \\
\end{tabular} & 4.68 & -0.60 & 0.011 \\
\hline Isfahan & 2.60 & 1.22 & 2.21 & -0.77 & 0.62 & 2.72 & 0.06 & 3.71 & -2.86 & \begin{tabular}{|l|}
-2.81 \\
\end{tabular} & 5.232 & -2.55 & N/A \\
\hline Kerman & 3.97 & 1.22 & 5.39 & -1.35 & 2.27 & 2.30 & 0 & 2.78 & -1.23 & \begin{tabular}{|l|}
0.50 \\
\end{tabular} & 3.12 & -0.80 & 0.017 \\
\hline Kermanshah & -0.55 & 1.22 & 0.33 & -1.04 & -2.12 & 3.61 & 0.04 & 3.92 & \begin{tabular}{|l|}
-0.62 \\
\end{tabular} & -0.81 & 4.99 & -1.37 & 0.035 \\
\hline Khuzestan & -0.44 & 0.24 & 0.95 & -4.09 & -2.49 & 4.32 & 0.61 & 3.24 & 0.51 & -0.88 & 4.91 & -0.81 & 0.086 \\
\hline Kohg \& B-Ahmad ${ }^{* *}$ & 0.80 & 0.73 & 1.99 & -1.47 & 0.90 & 3.94 & 0.54 & 2.01 & -1.26 & 0.10 & 4.96 & -1.02 & 0.010 \\
\hline Kordestan & -1.93 & 1.71 & -0.68 & -0.85 & -2.58 & 4.49 & 2.78 & 4.88 & -3.0 & 0.22 & 4.60 & -1.81 & 0.034 \\
\hline Lorestan & 0.01 & 1.71 & 0.12 & -0.75 & -2.27 & 3.58 & 0.24 & 5.04 & -1.35 & 0.40 & 4.62 & -0.51 & 0.044 \\
\hline Markazi & 0.72 & 0.73 & 1.11 & -0.68 & -0.07 & 2.95 & 0.44 & 4.37 & -1.71 & -1.28 & 4.74 & -1.21 & 0.033 \\
\hline Mazandaran & 0.91 & 1.71 & -0.68 & 0.17 & -2.04 & 3.27 & -1.80 & 2.63 & -0.46 & -1.55 & 4.34 & -3.75 & 0.072 \\
\hline North Khorasan & 1.46 & 1.71 & 2.54 & 0 & 1.25 & 2.87 & 0.01 & 3.28 & -0.93 & 0.57 & 4.27 & -3.56 & 0.023 \\
\hline Qazvin & 0.28 & 0.73 & 0.29 & -0.25 & -0.30 & 2.12 & 1.01 & 2.11 & -1.59 & -2.74 & 4.97 & -2.91 & 0.038 \\
\hline Qom & 0.58 & 0.73 & 1.64 & -0.75 & 0.15 & 3.05 & -0.03 & 4.45 & -3.10 & \begin{tabular}{|l|}
-3.09 \\
\end{tabular} & 4.82 & -3.07 & $\mathrm{~N} / \mathrm{A}$ \\
\hline Razavi Khorasan & 1.70 & 1.22 & 3.86 & 0.02 & 2.80 & 1.76 & -0.03 & 3.44 & -1.81 & 1.17 & 3.81 & -2.18 & $\mathrm{~N} / \mathrm{A}$ \\
\hline Semnan & 2.07 & 0.73 & 2.77 & -0.28 & 1.21 & 3.70 & -0.40 & 3.62 & -3.45 & -3.15 & 4.64 & -2.89 & $\mathrm{~N} / \mathrm{A}$ \\
\hline Sistan \& Baluch ${ }^{* * *}$ & 2.27 & 0.73 & 4.07 & -2.02 & 1.13 & 3.94 & 0.84 & 1.87 & -0.68 & 1.89 & 2.05 & 0.94 & 0.015 \\
\hline South Khorasan & 2.30 & 0.73 & 4.85 & -0.25 & 2.87 & 0.98 & 0.03 & 4.05 & \begin{tabular}{|l|}
-0.61 \\
\end{tabular} & 5.39 & 2.79 & 0.50 & 0.011 \\
\hline Tehran & 1.49 & 0.73 & 0.85 & -0.16 & -0.22 & 4.85 & -1.29 & 0.08 & -3.22 & -3.82 & 4.36 & -2.94 & $\mathrm{~N} / \mathrm{A}$ \\
\hline West Khorasan & -1.81 & 1.71 & 0.16 & -1.04 & 0.07 & 6.27 & 2.41 & 3.68 & -0.99 & 1.26 & 3.98 & -2.47 & 0.061 \\
\hline Yazd & 2.59 & 0.24 & 3.16 & -1.08 & 3.21 & 3.83 & -0.23 & 3.24 & -1.86 & 2.58 & 3.87 & -0.92 & 0.077 \\
\hline Zanjan & -1.43 & 1.22 & -0.23 & -0.69 & -2.04 & 3.79 & 1.39 & 3.67 & \begin{tabular}{|l|l|}
-0.89 \\
\end{tabular} & 0.089 & 4.86 & -2.71 & 0.020 \\
\hline \multicolumn{14}{|c|}{$\begin{array}{l}{ }^{*} \text { Chaharmahal and Bakhtiari } \\
{ }^{* *} \text { Kohgiluyeh and Boyer-Ahmad } \\
{ }^{* * *} \text { Sistan and Baluchestan }\end{array}$} \\
\hline
\end{tabular}


TABLE 22.3

Correlation and $\mathrm{P}$ value obtained from Pearson, Kendall, and Spearman, Pearson $\mathrm{P}$-Value: $\mathrm{P}-\mathrm{P}$ value; Kendall $\mathrm{P}$-value: $\mathrm{K}-\mathrm{P}$ value; Spearman $\mathrm{P}$-value: $\mathrm{S}-\mathrm{P}$ value.

\begin{tabular}{lcccccc}
\hline Indicators & Pearson & P-P value & Kendall & K-P value & Spearman & S-P value \\
Cloud Cover & -0.16 & 0.43 & -0.18 & 0.20 & -0.29 & 0.15 \\
ET & 0.13 & 0.53 & 0.19 & 0.24 & 0.24 & 0.25 \\
P & -0.35 & 0.08 & -0.28 & 0.05 & -0.39 & 0.05 \\
SP & 0.14 & 0.49 & 0.11 & 0.44 & 0.19 & 0.36 \\
SM & 0.06 & 0.74 & -0.05 & 0.70 & -0.06 & 0.77 \\
T & 0.10 & 0.62 & 0.03 & 0.80 & 0.06 & 0.77 \\
Wind & 0.15 & 0.47 & 0.12 & 0.41 & 0.16 & 0.43 \\
CO & 0.04 & 0.82 & 0.06 & 0.69 & 0.09 & 0.65 \\
HCHO & -0.03 & 0.88 & -0.10 & 0.50 & -0.12 & 0.54 \\
NO2 & -0.25 & 0.22 & -0.24 & 0.09 & -0.33 & 0.10 \\
O3 & 0.24 & 0.24 & 0.02 & 0.86 & 0.04 & 0.84 \\
SO2 & -0.22 & 0.27 & -0.19 & 0.19 & -0.31 & 0.13 \\
\hline
\end{tabular}

\subsection{Conclusion}

In this study, we examined the association between 12 spatial indicators obtained from satellite observations and COVID-19 cases in Iran using time-series analysis. Our findings indicated that changes of $\mathrm{SO} 2, \mathrm{NO} 2$, and precipitation were highly correlated with the number of COVID-19 cases. At least two correlation analysis methods including Pearson, Kendall, and Spearman and their P-values confirm that there was a relation between the three mentioned indicators and the number of COVID-19 cases. Changes of the effective indicators can be measured in other periods, so results may be useful for public health decision makers to mitigate the disease effects. As a future work, it is recommended to apply the methodology based on the number of COVID-19 deaths. Moreover, spatial modeling disease using these indicators is recommended as another topic for future studies.

\section{Acknowledgement}

The authors would like to thank the National Aeronautics and Space Administration (NASA) Land Processes Distributed Active Archive Center (DAAC, NASA's Goddard Earth Sciences Data and Information Services Center (GES DISC, National Oceanic and Atmospheric Administration (NOAA) National Weather Service (NWS) National Centers for Environmental Prediction (NCEP), NASA's Goddard Space Flight Center (GSFC), and European Space Agency (ESA) Copernicus, for providing temperature, evaporation, precipitation, surface pressure, soil moisture, wind speed, carbon monoxide (CO), nitrogen dioxide (NO2), sulphur dioxide (SO2), ozone (O3), formaldehyde $(\mathrm{HCHO})$, and cloud cover datasets.

\section{References}

[1] Najmul Haider, Alexei Yavlinsky, David Simons, Abdinasir Yusuf Osman, Francine Ntoumi, Alimuddin Zumla, and Richard Kock. Passengers' destinations from china: low risk of novel coronavirus (2019-ncov) transmission into africa and south america. Epidemiology \& Infection, 148, 2020. 
[2] Adrianna Murphy, Zhaleh Abdi, Iraj Harirchi, Martin McKee, and Elham Ahmadnezhad. Economic sanctions and iran's capacity to respond to covid-19. The Lancet Public Health, 5(5):e254, 2020.

[3] Zhu Yongjian, Xie Jingu, Huang Fengming, and Cao Liqing. Association between short-term exposure to air pollution and covid-19 infection: Evidence from china. Science of the Total Environment, page 138704, 2020.

[4] Yueling Ma, Yadong Zhao, Jiangtao Liu, Xiaotao He, Bo Wang, Shihua Fu, Jun Yan, Jingping Niu, Ji Zhou, and Bin Luo. Effects of temperature variation and humidity on the death of covid-19 in wuhan, china. Science of the Total Environment, page 138226, 2020.

[5] Abolfazl Mollalo, Abbas Alimohammadi, Mohammad Reza Shirzadi, and Mohammad Reza Malek. Geographic information system-based analysis of the spatial and spatio-temporal distribution of zoonotic cutaneous leishmaniasis in golestan province, north-east of iran. Zoonoses and Public Health, 62(1):18-28, 2014. doi: $10.1111 /$ zph.12109.

[6] Abolfazl Mollalo, Abbas Alimohammadi, and Mostafa Khoshabi. Spatial and spatio-temporal analysis of human brucellosis in iran. Transactions of The Royal Society of Tropical Medicine and Hygiene, 108(11): $721-728,2014$

[7] Abolfazl Mollalo, Jason Blackburn, Lillian Morris, and Gregory Glass. A 24-Year Exploratory Spatial Data Analysis of Lyme Disease Incidence Rate in Connecticut. Geospatial Health, 12(2), 2017. doi: 10.4081/gh. 2017.588 .

[8] Abolfazl Mollalo, Liang Mao, Parisa Rashidi, and Gregory E Glass. A gis-based artificial neural network model for spatial distribution of tuberculosis across the continental united states. International Journal of Environmental Research and Public Health, 16(1):157, 2019.

[9] Abolfazl Mollalo, Ali Sadeghian, Glenn D Israel, Parisa Rashidi, Aioub Sofizadeh, and Gregory E Glass. Machine learning approaches in gis-based ecological modeling of the sand fly phlebotomus papatasi, a vector of zoonotic cutaneous leishmaniasis in golestan province, iran. Acta Tropica, 188:187-194, 2018.

[10] Qian Liu, Dexuan Sha, Wei Liu, Paul Houser, Luyao Zhang, Ruizhi Hou, Hai Lan, Colin Flynn, Mingyue Lu, Tao Hu, et al. Spatiotemporal patterns of covid-19 impact on human activities and environment in mainland china using nighttime light and air quality data. Remote Sensing, 12(10):1576, 2020.

[11] Abolfazl Mollalo, Kiara M Rivera, and Behzad Vahedi. Artificial neural network modeling of novel coronavirus (covid-19) incidence rates across the continental united states. International Journal of Environmental Research and Public Health, 17(12):4204, 2020.

[12] Abolfazl Mollalo, Behzad Vahedi, and Kiara M Rivera. Gis-based spatial modeling of covid-19 incidence rate in the continental united states. Science of the Total Environment, page 138884, 2020.

[13] Noel Gorelick, Matt Hancher, Mike Dixon, Simon Ilyushchenko, David Thau, and Rebecca Moore. Google earth engine: Planetary-scale geospatial analysis for everyone. Remote Sensing of Environment, 2017. doi: 10.1016/j.rse.2017.06.031. URL https://doi.org/10.1016/j.rse.2017.06.031.

[14] Andrii Shelestov, Mykola Lavreniuk, Nataliia Kussul, Alexei Novikov, and Sergii Skakun. Exploring google earth engine platform for big data processing: Classification of multi-temporal satellite imagery for crop mapping. Frontiers in Earth Science, 5:17, 2017.

[15] Jalonne L White-Newsome, Shannon J Brines, Daniel G Brown, J Timothy Dvonch, Carina J Gronlund, Kai Zhang, Evan M Oswald, and Marie S O'Neill. Validating satellite-derived land surface temperature with in situ measurements: A public health perspective. Environmental Health Perspectives, 121(8):925-931, 2013.

[16] Fikre Enquselassie, ANNETTE J DOBSON, HILARY M ALEXANDER, and PAULA L STEELE. Seasons, temperature and coronary disease. International Journal of Epidemiology, 22(4):632-636, 1993.

[17] Z Wan, S Hook, and G Hulley. Mod11a1 modis/terra land surface temperature/emissivity daily 13 global $1 \mathrm{~km}$ sin grid v006. NASA EOSDIS Land Processes DAAC, 6, 2015.

[18] C Donald Ahrens. Essentials of meteorology: an invitation to the atmosphere. Cengage Learning, 2011.

[19] Ling-jun Li, WANG Ying, Qiang Zhang, YU Tong, ZHAO Yue, and JIN Jun. Spatial distribution of aerosol pollution based on modis data over beijing, china. Journal of Environmental Sciences, 19(8):955-960, 2007.

[20] Lei Lu, Tingjun Zhang, Tiejun Wang, and Xiaoming Zhou. Evaluation of collection-6 modis land surface temperature product using multi-year ground measurements in an arid area of northwest china. Remote Sensing, 10(11):1852, 2018.

[21] Thanh Noi Phan and Martin Kappas. Application of modis land surface temperature data: a systematic literature review and analysis. Journal of Applied Remote Sensing, 12(4):041501, 2018.

[22] Steven W Running, Qiaozhen Mu, Maosheng Zhao, and Alvaro Moreno. Modis global terrestrial evapotranspiration (et) product (nasa mod16a2/a3) nasa earth observing system modis land algorithm. NASA: Washington, DC, USA, 2017. 
[23] Dario Autovino, Mario Minacapilli, and Giuseppe Provenzano. Modelling bulk surface resistance by modis data and assessment of mod16a2 evapotranspiration product in an irrigation district of southern italy. Agricultural Water Management, 167:86-94, 2016.

[24] Marjolein FA Vogels, Steven M de Jong, Geert Sterk, Niko Wanders, Marc FP Bierkens, and Elisabeth A Addink. An object-based image analysis approach to assess irrigation-water consumption from modis products in ethiopia. International Journal of Applied Earth Observation and Geoinformation, 88:102067, 2020.

[25] Andreas Tornevi, Olof Bergstedt, and Bertil Forsberg. Precipitation effects on microbial pollution in a river: lag structures and seasonal effect modification. PloS One, 9(5):e98546, 2014.

[26] Arthur Y Hou, Ramesh K Kakar, Steven Neeck, Ardeshir A Azarbarzin, Christian D Kummerow, Masahiro Kojima, Riko Oki, Kenji Nakamura, and Toshio Iguchi. The global precipitation measurement mission. Bulletin of the American Meteorological Society, 95(5):701-722, 2014.

[27] George J Huffman, David T Bolvin, Dan Braithwaite, Kuolin Hsu, Robert Joyce, Pingping Xie, and Soo-Hyun Yoo. Nasa global precipitation measurement (gpm) integrated multi-satellite retrievals for gpm (imerg). Algorithm Theoretical Basis Document (ATBD) Version, 4:26, 2015.

[28] Ji-Hye Kim, Mi-Lim Ou, Jun-Dong Park, Kenneth R Morris, Mathew R Schwaller, and David B Wolff. Global precipitation measurement (gpm) ground validation (gv) prototype in the korean peninsula. Journal of Atmospheric and Oceanic Technology, 31(9):1902-1921, 2014.

[29] Kiyoung Kim, Jongmin Park, Jongjin Baik, and Minha Choi. Evaluation of topographical and seasonal feature using gpm imerg and trmm 3b42 over far-east asia. Atmospheric Research, 187:95-105, 2017.

[30] Luiz Octavio Fabricio dos Santos, Carlos Alexandre Santos Querino, Juliane Kayse Albuquerque da Silva Querino, Altemar Lopes Pedreira Junior, Aryanne Resende de Melo Moura, Nadja Gomes Machado, and Marcelo Sacardi Biudes. Validation of rainfall data estimated by gpm satellite on southern amazon region. Revista Ambiente \&S Água, 14(1), 2019.

[31] Wei Sun, Yonghua Sun, Xiaojuan Li, Tao Wang, Yanbing Wang, Qi Qiu, and Zhitian Deng. Evaluation and correction of gpm imerg precipitation products over the capital circle in northeast china at multiple spatiotemporal scales. Advances in Meteorology, 2018, 2018.

[32] Uta Ferrari, Teresa Exner, Eva R Wanka, Christoph Bergemann, Julian Meyer-Arnek, Beate Hildenbrand, Amanda Tufman, Christian Heumann, Rudolf M Huber, Michael Bittner, et al. Influence of air pressure, humidity, solar radiation, temperature, and wind speed on ambulatory visits due to chronic obstructive pulmonary disease in bavaria, germany. International Journal of Biometeorology, 56(1):137-143, 2012.

[33] Suranjana Saha, Shrinivas Moorthi, Hua-Lu Pan, Xingren Wu, Jiande Wang, Sudhir Nadiga, Patrick Tripp, Robert Kistler, John Woollen, David Behringer, et al. The ncep climate forecast system reanalysis. Bulletin of the American Meteorological Society, 91(8):1015-1058, 2010.

[34] Dong-Ik Kim and Dawei Han. Comparative study on long term climate data sources over south korea. Journal of Water and Climate Change, 10(3):504-523, 2019

[35] Tiina Nygård, Timo Vihma, Gerit Birnbaum, Jörg Hartmann, John King, Tom Lachlan-Cope, Russell Ladkin, Christof Lüpkes, and Alexandra Weiss. Validation of eight atmospheric reanalyses in the antarctic peninsula region. Quarterly Journal of the Royal Meteorological Society, 142(695):684-692, 2016.

[36] Dara Entekhabi, Simon Yueh, Peggy E O'Neill, Kent H Kellogg, Angela Allen, Rajat Bindlish, Molly Brown, Steven Chan, Andreas Colliander, Wade T Crow, et al. Smap handbook-soil moisture active passive: Mapping soil moisture and freeze/thaw from space. 2014.

[37] Nereida Rodriguez-Alvarez, Sidharth Misra, and Mary Morris. The polarimetric sensitivity of smap-reflectometry signals to crop growth in the us corn belt. Remote Sensing, 12(6):1007, 2020.

[38] Nazmus Sazib, Iliana Mladenova, and John Bolten. Leveraging the google earth engine for drought assessment using global soil moisture data. Remote Sensing, 10(8):1265, 2018

[39] Huan Wu, John S Kimball, Naijun Zhou, Lorenzo Alfieri, Lifeng Luo, Jinyang Du, and Zhijun Huang. Evaluation of real-time global flood modeling with satellite surface inundation observations from smap. Remote Sensing of Environment, 233:111360, 2019.

[40] Chengwei Li, Hui Lu, Kun Yang, Menglei Han, Jonathon S Wright, Yingying Chen, Le Yu, Shiming $\mathrm{Xu}$, Xiaomeng Huang, and Wei Gong. The evaluation of smap enhanced soil moisture products using high-resolution model simulations and in-situ observations on the tibetan plateau. Remote Sensing, 10 (4):535, 2018.

[41] BG Mousa and Hong Shu. Spatial evaluation and assimilation of smap, smos, and ascat satellite soil moisture products over africa using statistical techniques. Earth and Space Science, 7(1):e2019EA000841, 2020.

[42] Josh M Colston, Tahmeed Ahmed, Cloupas Mahopo, Gagandeep Kang, Margaret Kosek, Francisco de Sousa Junior, Prakash Sunder Shrestha, Erling Svensen, Ali Turab, Benjamin Zaitchik, et al. Evaluating meteorological data from weather stations, and from satellites and global models for a multi-site epidemiological study. Environmental Research, 165:91-109, 2018. 
[43] Wen Wang, Wei Cui, Xiaoju Wang, and Xi Chen. Evaluation of gldas-1 and gldas-2 forcing data and noah model simulations over china at the monthly scale. Journal of Hydrometeorology, 17(11):2815-2833, 2016.

[44] Elie El Khoury, Elsy Ibrahim, and Sophia Ghanimeh. A look at the relationship between tropospheric nitrogen dioxide and aerosol optical thickness over lebanon using spaceborne data of the copernicus programme. In 2019 Fourth International Conference on Advances in Computational Tools for Engineering Applications (ACTEA), pages 1-6. IEEE, 2019.

[45] Hichem Omrani, Bilel Omrani, Benoit Parmentier, and Marco Helbich. Spatio-temporal data on the air pollutant nitrogen dioxide derived from sentinel satellite for france. Data in Brief, 28:105089, 2020.

[46] S Running, Q Mu, and M Zhao. Mod16a2 modis/terra net evapotranspiration 8-day 14 global 500m sin grid v006. NASA EOSDIS Land Processes DAAC, 6, 2017.

[47] GJ Huffman, EF Stocker, DT Bolvin, EJ Nelkin, and T Jackson. Gpm imerg final precipitation 13 half hourly 0.1 degree x 0.1 degree v06, greenbelt, md, goddard earth sciences data and information services center (ges disc), 2019.

[48] PE O’Neill, S Chan, EG Njoku, T Jackson, and R Bindlish. Smap 13 radiometer global daily 36 km ease-grid soil moisture, version 4, boulder, colorado usa, nasa national snow and ice data center distributed active archive center, 2016.

[49] Matthew Rodell, PR Houser, UEA Jambor, J Gottschalck, K Mitchell, C-J Meng, K Arsenault, B Cosgrove, J Radakovich, M Bosilovich, et al. The global land data assimilation system. Bulletin of the American Meteorological Society, 85(3):381-394, 2004.

[50] Hossein Ahani, Mehrzad Kherad, Mohammad Reza Kousari, Mehdi Rezaeian-Zadeh, Mohammad Amin Karampour, Faezeh Ejraee, and Saeedeh Kamali. An investigation of trends in precipitation volume for the last three decades in different regions of fars province, iran. Theoretical and Applied Climatology, 109 $(3-4): 361-382,2012$.

[51] Mohammad Reza Kousari, Mohammad Amin Asadi Zarch, Hossein Ahani, and Hemila Hakimelahi. A survey of temporal and spatial reference crop evapotranspiration trends in iran from 1960 to 2005. Climatic Change, 120(1-2):277-298, 2013.

[52] Jean Dickinson Gibbons and Subhabrata Chakraborti. Nonparametric Statistical Inference: Revised and Expanded. CRC press, 2014

[53] Jacob Benesty, Jingdong Chen, Yiteng Huang, and Israel Cohen. Pearson correlation coefficient. In Noise reduction in speech processing, pages 1-4. Springer, 2009.

[54] Douglas G Bonett and Thomas A Wright. Sample size requirements for estimating pearson, kendall and spearman correlations. Psychometrika, 65(1):23-28, 2000.

[55] H Abdi. The kendall rank correlation coefficient. encyclopaedia of measurement and statistics (pp. 508-510), 2007. 